\title{
Quantum shape phase transitions from spherical to deformed for Bose-Fermi systems: the effect of the odd particle around the critical point
}

\author{
M. Böyükata ${ }^{1,2, a}$, C. E. Alonso ${ }^{3}$, J. M. Arias ${ }^{3}$, L. Fortunato ${ }^{4}$ and A. Vitturi ${ }^{4}$ \\ ${ }^{1}$ Institüt für Kernphysik, Universität zu Köln, D-50937 Köln, Germany \\ ${ }^{2}$ Department of Elementary Science Education, Faculty of Education Çanakkale Onsekiz Mart University, \\ TR-17100 Çanakkale, Turkey \\ ${ }^{3}$ Departamento de Fisica Atomica, Molecular y Nuclear, Universidad de Sevilla, ES-41080 Sevilla, Spain \\ ${ }^{4}$ Dipartimento di Fisica e Astronomia "G. Galilei", Università di Padova and INFN, Sezione di Padova, I-35131 \\ Padova, Italy
}

\begin{abstract}
Quantum phase transitions in odd-nuclei are investigated within the framework of the interacting boson-fermion model with a description based on the concept of intrinsic states. We consider the case of a single $j=9 / 2$ odd-particle coupled to an even-even boson core that performs a transition from spherical to deformed prolate and to deformed gamma-unstable shapes varying a control parameter in the boson Hamiltonian. The effect of the coupling of the odd particle to this core is discussed along the shape transition and, in particular, at the critical point.
\end{abstract}

\section{Introduction}

Quantum Phase Transitions (QPT) have been one of the top subjects in nuclear physics, especially during the last decade. Phase transitions studies have been mostly performed on even-even systems [1] within the interacting boson model (IBM) [2] and also recently in bose-fermi systems [3-11] within the interacting boson fermion model (IBFM) [12]. IBM has three dynamical symmetries denoted by $\mathrm{U}(5), \mathrm{SU}(3)$ and $\mathrm{O}(6)$. Each dynamical symmetry is related to a different shape: spherical (U(5)), deformed axial prolate (SU(3)) and deformed gamma-unstable $(\mathrm{O}(6))$. The QPT from U(5) to SU(3) is first-order while the transition $\mathrm{U}(5)$ to $\mathrm{O}(6)$ is second-order (continuous). Similar shape QPT's are found in the context of the Bohr Hamiltonian. Critical point symmetries have been proposed to model their critical points: X(5), from spherical to axially deformed (first order), and E(5), from spherical to deformed gamma-unstable (second order) [13].

The purpose of this work is to see the effect of the coupling of a single fermion to a boson core that performs a transition from spherical to axially prolate shapes, in particular, at the critical point and its around. We, therefore, intend to explain how the coupling of the single fermion in orbit of definite angular momentum $\mathrm{j}$ modifies the geometry imposed by the boson core performing the transition and to describe the behavior of each individual coupled state of single $\mathrm{j}$ at the transitional region. The odd particle effects along $\mathrm{U}(5) \rightarrow \mathrm{SU}(3)$ and $\mathrm{U}(5) \rightarrow \mathrm{O}(6)$ transitions are compared.

\footnotetext{
a e-mail: boyukata@comu.edu.tr
} 


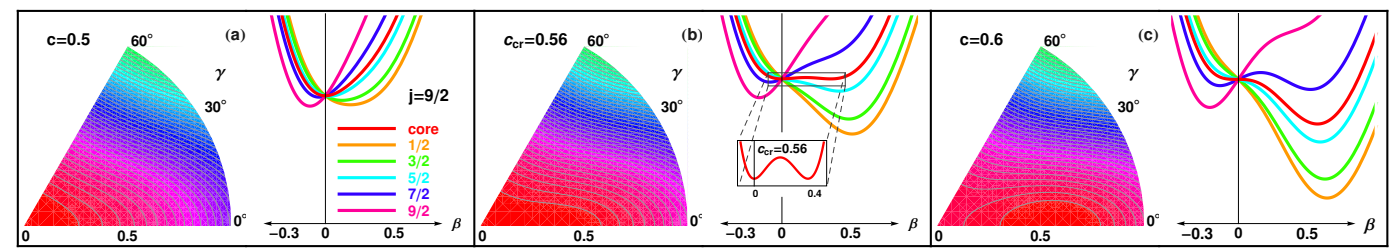

Figure 1. The effect of odd particle on the core around the U(5)-SU(3) critical point. Energy surfaces at $c=0.5$ in (a), at the critical point $c_{c r}=0.56$ in (b), at $c=0.6$ in (c). Contour plots in the $\beta-\gamma$ plane are for the even-even core with five bosons. Cuts of the energy surfaces as a function of $\beta\left(\gamma=0^{\circ}\right)$ are for the odd-even system for different states labeled by $K$ with different colors, as defined in (a), for each magnetic component of $j=9 / 2$.

\section{Intrinsic Frame Formalism of Interacting Boson Fermion Model}

Quantum shape phase transitions for bose-fermi systems can be described within the framework of the intrinsic frame formalism of the interacting boson-fermion model. The IBFM Hamiltonian including the boson part, the fermion part and the boson-fermion interaction is generally written as

$$
H_{B F}=H_{B}+H_{F}+V_{B F} .
$$

The $\mathrm{H}_{B}$ boson Hamiltonian is here parametrized as

$$
H_{B}=(1-c) \hat{n}_{d}-\frac{c}{4 N} \hat{Q}_{B}^{\chi} \cdot \hat{Q}_{B}^{\chi}
$$

where $N$ is the total boson number, $c$ is the control parameter, $\hat{n}_{d}$ is the d-boson number operator given by $\hat{n}_{d}=\sum_{\mu} d_{\mu}^{\dagger} d_{\mu}$ and $Q_{B}^{\chi}$ is the boson quadrupole operator $\hat{Q}_{B}^{\chi}=\left(s^{\dagger} \times \tilde{d}+d^{\dagger} \times \tilde{s}\right)^{(2)}+\chi\left(d^{\dagger} \times \tilde{d}\right)^{(2)}$. The boson-fermion Hamiltonian can be correspondingly written as

$$
H_{B F}=(1-c) \hat{n}_{d}-\frac{c}{4 N} \hat{Q}_{B F}^{\chi} \cdot \hat{Q}_{B F}^{\chi},
$$

where the quadrupole operator for the odd-even system is $\hat{Q}_{B F}^{\chi}=\hat{Q}_{B}^{\chi}+\hat{q}_{F}$ and here $\hat{q}_{F}$ is the fermion quadrupole operator that is given by $\hat{q}_{F}=\left(a_{j}^{\dagger} \times \tilde{a}_{j}\right)^{(2)}$. The $H_{F}$ fermion part in Eq. (1) is a constant for the single-j shell case and the boson-fermion interaction obtained from Eq. (3) is $\hat{V}_{B F}=-\frac{c}{2 N} \hat{Q}_{B}^{\chi} \cdot \hat{q}_{F}$.

The intrinsic frame formalism can be used to describe phase transitions with the potential energy surfaces obtained from the boson Hamiltonian (2). The intrinsic state for the ground state $(g s)$ band is written as a function of the shape variables $(\beta, \gamma)$

$$
\Phi_{g s}(\beta, \gamma)=\frac{1}{\sqrt{N !}}\left[\frac{1}{\sqrt{1+\beta^{2}}}\left(s^{\dagger}+\beta \cos \gamma d_{0}^{\dagger}+\frac{\beta}{\sqrt{2}} \sin \gamma\left(d_{2}^{\dagger}+d_{-2}^{\dagger}\right)\right)\right]^{N}|0\rangle
$$

where $|0\rangle$ is the boson vacuum. The ground state energy surface is obtained by calculating the expectation value of the boson Hamiltonian in the intrinsic state $E_{g s}(\beta, \gamma)=\left\langle\Phi_{g s}(\beta, \gamma)\left|H_{B}\right| \Phi_{g s}(\beta, \gamma)\right\rangle$. Intrinsic frame states for the mixed boson-fermion system can be constructed by coupling the odd single-particle states to the intrinsic states of the even core. The lowest states of the odd nucleus are expected to originate from this coupling to the intrinsic ground state $\Phi_{g s}(\beta, \gamma)$. To obtain them, we first construct the coupled states $\Psi_{j K}(\beta, \gamma)=\Phi_{g s}(\beta, \gamma) \otimes|j K\rangle$ and then diagonalize the total boson-fermion Hamiltonian in this basis. Here, $K$ is the magnetic component of the total angular momentum $j$. 


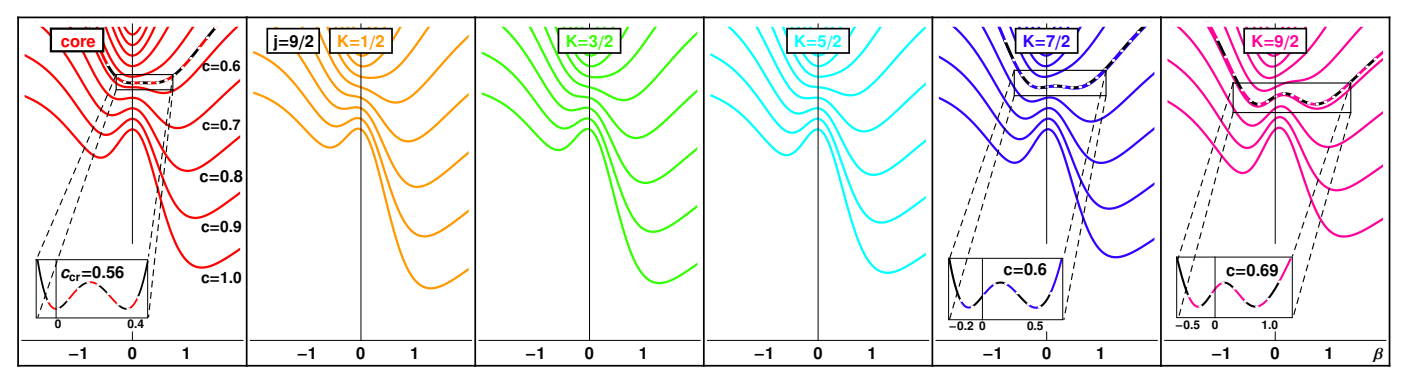

Figure 2. Even-even (red color) and odd-even energy surfaces as a function of $\beta$. The control parameter $c$ is changed from 0 to $l$ in the Hamiltonian (from spherical to prolate shape, $\chi=-\sqrt{7} / 2$ ).

\section{Results and Conclusions}

In the IBM Hamiltonian (2), the $\mathrm{U}(5)$ limit is obtained with $c=0$, while $c=1$ gives pure $\mathrm{O}(6)$ and SU(3) symmetries for $\chi=0$ and $\chi=-\sqrt{7} / 2$, respectively. The first-order transition appears at $c_{c r}=16 N /(34 N-27)$ in between $\mathrm{U}(5)-\mathrm{SU}(3)$ [7] while the second-order transition appears at $c_{c r}=N /(2 N-2)$ in between $\mathrm{U}(5)-\mathrm{O}(6)$ [8] for the even-even boson system. In the case of 5 bosons, the critical point of the first-order transition is $c_{c r}=0.56$ while it is 0.625 for the second-order transition. The effect of the coupling of an odd particle to a $\mathrm{O}(6)$ boson core was discussed in detail in Ref. [8]. According to the classical analysis of both cases for $j=11 / 2$ performed by Petrellis et al. [10], the phase transition is washed out for states with $K=1 / 2,3 / 2,5 / 2$ but other states with larger $K$ $(7 / 2,9 / 2,11 / 2)$ show first order transition and the phase transition is dramatically changed by the exchange term. However, in their analysis in [11], states states with larger $K$ show oblate shape then jump to prolate and the phase transition is also washed out for $K=11 / 2$ with larger quadrupole coupling constant $\Gamma$. Here we focus on the effect of the coupling of an odd particle with $j=9 / 2$ to an even-even core that transits from spherical to prolate shapes, particularly around the critical point.

Therefore, we assume as examples for the control parameter the values $c=0.5, c_{c r}=0.56, c=0.6$ in the Hamiltonian (2) and the expectation value of the boson Hamiltonian $E_{g s}(\beta, \gamma)$ in the intrinsic state (4) is calculated to obtain the ground state energy surface. Then the odd $j=9 / 2$ particle is coupled to the core using the boson-fermion interaction $V_{B F}$. The energy surfaces for the boson core as a function of the deformation parameters $(\beta, \gamma)$ are plotted around the critical point in Fig. 1. The behaviour of the odd-even energy surfaces as a function of $\beta$ is illustrated around critical point in the left side of (a), (b), (c) panels of Fig. 1. In these panels, the boson core is denoted with red color as a reference. It is clearly seen in Fig. 1(b) that the even-even surface has two minima at $c_{c r}=0.56$. The even-even system is spherical before this point then it jumps to prolate. The intrinsic states of the odd-even system prefer prolate or oblate shapes while the boson core is spherical in Fig. 1(a), prolate in Fig. 1(c) or located at critical point in Fig. 1(b).

The evolution of the energy surfaces along the shape phase transition for the boson core with five magnetic components $K$ is illustrated in Fig. 2. States with $K=1 / 2,3 / 2,5 / 2$ always favor prolate shapes, while states with $K=7 / 2,9 / 2$ are oblate up to $c_{7 / 2} \simeq 0.6$ and $c_{9 / 2} \simeq 0.69$. After these points, they suddenly change to prolate shapes. Therefore we can suggest that both states, $K=7 / 2,9 / 2$, show first order transitions, since they have two absolute minima as seen in the inserts in the last two panels of Fig. 2.

When moving towards the critical point the states with larger $K$ values move to their preferred oblate deformation, while the states with smaller $K$ values are prolate as seen in left side of Fig. 3 . After the bosonic critical point each oblate state will shift to prolate shape at some specific value of the control parameter. In the pure prolate deformed region the dominant action of the strongly prolate core drives all odd states into the prolate side and they have approximately the same $\beta_{\min }$ as the prolate 


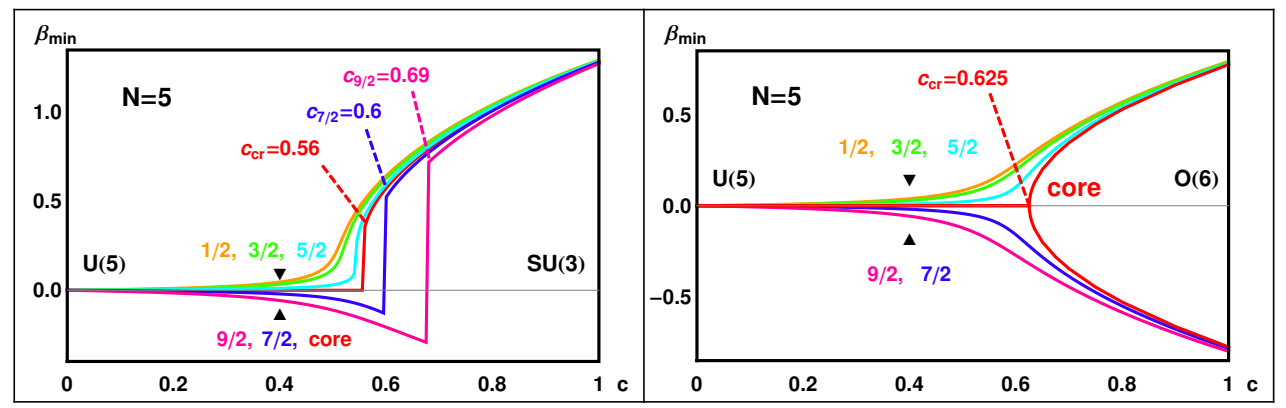

Figure 3. Evolution of the equilibrium deformation parameter corresponding to the different $\mathrm{K}$ states in the odd-even system as a function of the control parameter $c$ along the spherical to deformed region: the transition from $\mathrm{U}(5)$ to $\mathrm{SU}(3)$ (in left panel) and the transition from $\mathrm{U}(5)$ to $\mathrm{O}(6)$ (right panel from Ref. [8]). Positive and negative values of $\beta_{\min }$ indicate prolate and oblate shapes, respectively.

core. For comparison, we also show the case of $\mathrm{U}(5)$ to $\mathrm{O}(6)$ transition in the right panel of Fig. 3. The states with low $K$ values always prefer to be prolate, while the states with high $K$ values always prefer oblate shapes and hence phase transition for odd states with all $K$ is washed out in this case.

In these proceedings, the coupling of a single $j=9 / 2$ particle to a boson core that undergoes a transition from spherical to prolate has been investigated and results are compared with our previous work [8]. Forthcoming studies will be focused on the effect of the single particle (for different angular momenta, $j$ is changing from $3 / 2$ to $13 / 2$ ) coupling to the even-even core performing not only the transition from spherical to prolate $(U(5)-S U(3))$ but also from spherical to oblate $(U(5)-\overline{S U(3)})$ and from prolate to oblate $(S U(3)-\overline{S U(3)})$.

\section{Acknowledgments}

This work is supported by the Scientific and Technical Research Council of Turkey (TÜBİTAK) BIDEB-2219 and Spanish MEC project FIS2011-28738-C02-01.

\section{References}

[1] P. Cejnar, J. Jolie and R. F. Casten, Rev. Mod. Phys. 82, 2155 (2010)

[2] F. Iachello and A. Arima, The Interacting Boson Model, Cambridge University Press (1987)

[3] J. Jolie, S. Heinze, P. Van Isacker and R. F. Casten, Phys. Rev. C 70, 011305(R) (2004)

[4] F. Iachello, Phys. Rev. Lett. 95, 052503 (2005)

[5] C. E. Alonso, J. M. Arias, L. Fortunato and A. Vitturi, Phys. Rev. C 72, 061302(R) (2005)

[6] C. E. Alonso, J. M. Arias and A. Vitturi, Phys. Rev. Lett. 98, 052501 (2007); Phys. Rev. C 75, 064316 (2007)

[7] C. E. Alonso, J. M. Arias, L. Fortunato and A. Vitturi, Phys. Rev. C 79, 014306 (2009)

[8] M. Böyükata, C. A. Alonso, J. M. Arias, L. Fortunato and A. Vitturi, Phys. Rev. C 82, 014317 (2010)

[9] L. Fortunato, C. A. Alonso, J. M. Arias, L. Fortunato M. Böyükata and A. Vitturi, Int. J. Mod. Phys. E 20, 207 (2011)

[10] D. Petrellis, A. Leviatan, and F. Iachello, Ann. of Phys. 326, 926 (2011)

[11] F. Iachello, A. Leviatan, and D. Petrellis, Phys. Lett. B 705, 379 (2011)

[12] F. Iachello and P. Van Isacker, The Interacting Boson-Fermion Model, Cambridge University Press (1991)

[13] F. Iachello, Phys. Rev. Lett. 85, 3580 (2000); Phys. Rev. Lett. 87, 052502 (2001)

[14] M. Böyükata, C. A. Alonso, J. M. Arias, L. Fortunato and A. Vitturi, in progress (2013) 